\title{
Mosaic Evaluation: An Efficient and Robust Method based on Maximum Information Retrieval
}

\author{
Kantilal P.Rane \\ GF's GCOE, \\ Jalgaon (INDIA)
}

\author{
Mayur B. \\ Limbachiya \\ GF's GCOE, \\ Jalgaon (INDIA)
}

\author{
Shrirang S.Pandit \\ GF's GCOE, \\ Jalgaon (INDIA)
}

\author{
Sunil G. Bhirud \\ VJTI, \\ Mumbai (INDIA)
}

\begin{abstract}
Performance Evaluation Process for video mosaic algorithms is developed on the basis of maximum information retrieval through closeness and residue between the original input images/ actual frames and the estimated images/frames from mosaic image. This evaluation method can be applicable to image as well as video mosaicing methods. Estimation of original input images/video frames and maximum information retrieval in terms of closeness/residue are the major steps involved in it. Without specific design of standard database, this method evaluates the mosaics in reference with the information in original input images/frames through a unique and single valued metric. Problems in case of mosaicing in complex condition like nonlinear vertical distortions and geometrical distortions in image and video capturing are discussed. Performance results are tested and compared with different mosaic images from different mosaic algorithms presented before.
\end{abstract}

\section{Keywords}

Mosaic evaluation; Image mosaic; Strip search.

\section{INTRODUCTION}

Mosaic evaluations based on human judgment and personal analysis has been performed in early days. In latter days, image blurring [1] like ad hoc measures have been used but overall performance is not satisfactory. Uses of ground truth information of standard data sets were developed [2-6] during next days in various methods which included the various measuring metrics in 1 to 4-5 numbers. Estimation of standard data sets was a crucial at that time but very difficult task in these methods to cover real world data. Latter, very few methods [7-8] were introduced with no use of standard data set whose results are also satisfactory.

Various parameter's evaluation and various system's ranking based on mosaic creation were proposed in literature like ranking of orientation tracking systems [9], ranking of electrooptical (EO) systems [10], Radiographic Quality [11], ranking of radiographic digital system [12], quality of size, shape and position of the image layer in radiographic panoramic images [13], quality of video compression [14] and ranking of tracking methods [15].

Based on the various methods proposed in literature, various requirements in evaluation methodologies are:

1) To find the single and unique valued metric with high speed and reliable operations so that ranking of mosaic method and quality evaluation of mosaics is feasible with real world data.

2) To develop the evaluation method for video mosaic to handle vertical distortions while propagating in horizontal direction.
3) To work without use of standard and reference databases for making the system computationally efficient.

4) To combine the evaluation of image mosaicing and video mosaicing.

Mosaic evaluation should provide a measurable value for similarity between mosaic image and the input images/frames. It is required to determine some evaluative terms so that it will provide unique, accurate values for mosaic results. Problem is to develop mathematical model for evaluative terms. Model should be robust and should work on any image/video mosaicing technique.

There is very less work reported on quantitative mosaic evaluation methodology without use of standard database. We tried to develop the evaluation method which is based on the information retrieval from the original images/frames. Depending on the information available in mosaic image, performance of image/video mosaic can be measured. Suitable steps of mosaic image evaluation includes,

1) Finding the maximum information areas (of original image/frame size) within the mosaic image, with respective to all the original image/frames of video which may be called Estimation of Video Frames/Input Images.

2) Combining the information present within all the estimated images/frames of video, this is suggested to be calculated in terms of percentage closeness and percentage residue.

In case of image mosaicing, image transformation and image warping are generally wide, but it is harmful if images to be stitched are very large, which may lead to complex nature. Due to this one to one pixel based evaluation is even suitable in case of image mosaicing which indirectly can evaluate complexity. If the transformation is wide, complexity is more and should be indicated by the degradation of the ranking of mosaic method. So, same evaluation method can be applied to image as well as video mosaicing.

Evaluation of nonlinear vertically distorted mosaic image from image stitching and video stitching may have different concept. In video mosaicing, overlapped portion is larger than that of in image mosaicing. So, with nonlinear vertical distortion, chances of direct correspondence of frames with mosaic in video mosaicing are less with respective to that of in image mosaicing. This indicates less evaluation performances in video mosaicing with respective to image mosaicing.

Image mosaicing are generally used where geometrical distortions are more instead of video mosaicing. So, with geometrical distortions, evaluation performance of video mosaicing is exactly reverse (more) than that of in image mosaicing. 
Problem of evaluation of image mosaicing in geometrical distortion is already recovered by many authors with the use of registration based evaluation [2-4]. Only solution is required to handle the problem of evaluation of video mosaicing with nonlinear distortion.

\section{METHODOLOGY}

Mosaic evaluation technique for image as well as video mosaicing can includes two steps as,

1) Estimation of Input Images/Video Frames and

2) Closeness and residue as a performance measure.

\subsection{Estimation of Input Images/Video Frames}

Final mosaic generated may be enlarged mosaic image of height M1 and width L1. It is required to be resized into height $\mathrm{M}$ of the original image /frame and width $\mathrm{L}$ with proper aspect ratio so that maximum information can be retrieved from it. Similarities [16] between original images/frames and all possible striped images/frames from mosaic image are calculated by using equation (1).

$\operatorname{Sim}_{i}(j)=$

Similarity $_{j=1:(L-N)}\left(f_{i}, s_{j}(x, y, z)\right)$, for $i^{\text {th }}$ image/fram

Where,

$f_{i}$ is $i^{\text {th }}$ original image/frame,

$s_{j}$ is $j^{\text {th }}$ strip from mosaic image, $N$ is the width of original image/frame, $x=1$ to $M, y=j$ to $(j+N)$ and $z=1$ to 3

Strip identifier $j$ of maximum similarity $\operatorname{Sim}_{i}(j)$ of $i^{\text {th }}$ original image/frame indicate location (node) of estimated image/frame and is enlisted as $\operatorname{ind} x(i)$.

Estimated image/frame is modeled by equation (2) as,

$$
\widehat{f}_{i}=s_{\text {ind }(i)}(x, y, z), \text { for all, } x=1 \text { to } M, y=
$$

1 to $N, z=1$ to 3

It is required to estimate number of images/frames $\widehat{f}_{i}$ and their nodes list $i n d x(i)$ for all $n$ original images/frames on account to measure the performance.

\subsection{Performance Measure: Closeness and Residue}

Performance of mosaic image is evaluated in terms of Overall Percentage Closeness (OPC) or Overall Percentage Residue (OPR) between original and estimated images/frames as given in equation (6) and equation (7) because of possibility of maximum information retrieval using it. Closeness $C_{i}$ between $f_{i}$ and $\widehat{f}_{i}$ is considered as a count of number of black pixels within the difference image of $f_{i}$ and $\widehat{f}_{i}$ as black pixel is a difference of same pixels of any grey value and can be calculated by histogram analysis of difference image.

Difference image $\left(d_{i}\right)$ is given in equation (3).

$d_{i}=f_{i}-\widehat{f}_{i}$, for all images $/$ frames of $i=1$ to $n$,

Where, $n$ is the total number of original image/frames.
As $1^{\text {st }}$ value in histogram of difference image (hist) is a count of zero grey level pixels (black pixels), Closeness $\left(C_{i}\right)$ can be stated as,

$C_{i}=\operatorname{hist}(1)$

Overall closeness over all the frames of video is given as,

$O c=\left(\frac{1}{n}\right) * \sum_{i=1: n} C_{i}$

Overall Percentage Closeness (OPC) and Overall Percentage Residue (OPR) are calculated as,

$O P C=\left(\frac{O c}{(N * M)}\right) * 100$

Where, $M$ is hight and $\mathrm{N}$ is width of original frame and

$O P R=1-O P C$

More closeness of original image/frame $f_{i}$ and estimated image/frame $\widehat{f}_{i}$ indicates less $O P R$.

Geometrical distortions in case of image and video mosaicing can be also evaluated with OPC and OPR. As video traversing in any direction doesn't involve large geometrical distortions, there is no need to think on the solution in the case of video mosaicing. OPC and OPR are the suitable measure for wide geometrical distortion because of complexity criteria to create it. With the use of proposed measures, mosaic created with nonlinear distortions in process of image mosaicing can be evaluated accurately because of wide overlapped portions between consecutive input images. But nonlinear distortions in video mosaicing may provide wrong evaluation due to the global and local vertical deviations as shown in Fig. 1 and Fig. 3 respectively. Evaluation of mosaic with global and local vertical deviations created from nonlinear vertically distorted video can be accurate with proposed measures by cropping the frames along top and bottom sides. It minimizes the errors in direct matching of cropped frames (Fig. 2 and Fig. 3) using OPC/OPR than that of full frames (Fig. 1).

\section{Experimental Results}

Various mosaic images (Fig.4 to Fig. 8) are evaluated and their evaluation results are presented in Table 1 . It shows that most of the videos are having $50-60 \%$ closeness to its mosaic images. Mosaic performance (in percentage OPC) from Beach View video with Wexler's method [18] as indicated in Table 1 is compared with the performance from Strip Search Algorithms [16] method.

Poor evaluations are estimated from the mosaic images created with full frames when we used the Strip Search Algorithm and Benedict's method for video mosaicing due to the nonlinear vertical distortions, even the mosaic images are visualized well. Cropped frames (Fig. 2 and Fig. 3) are applied for evaluation with both the mosaic creation methods and found the drastic change in its performances. Also performance with mosaic created with nonlinear vertical distortions is checked using image mosaicing method (Andrew's method) as shown in Fig. 9, and found the results satisfactory. 


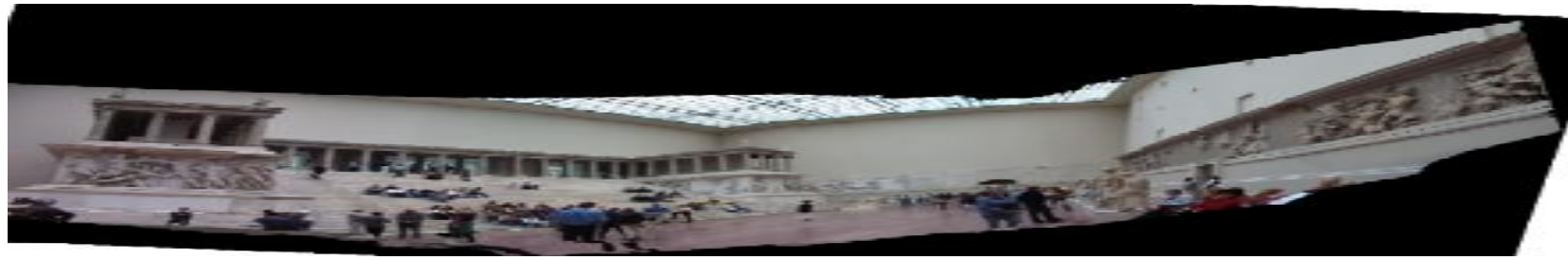

Fig. 1:Mosaic1: Global vertical deviation in mosaic created from nonlinear distorted video by Benedict et. al [17]showing direct matching process of full frames

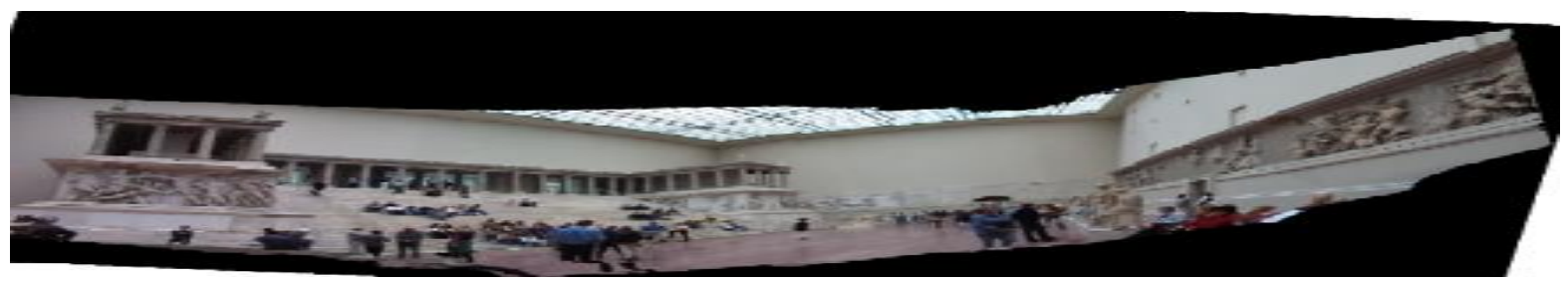

Fig. 2:Mosaic1: Global vertical deviation in mosaic created from nonlinear distorted video by Benedict et. al showing direct matching process of cropped frames

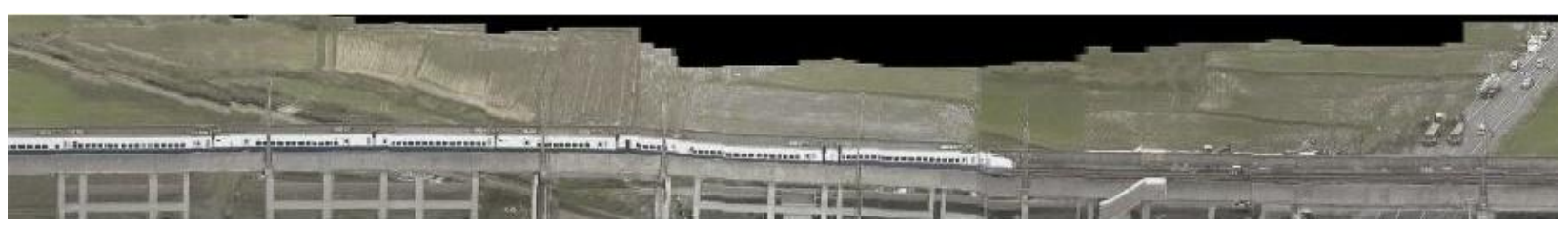

Fig. 3:Mosaic2: Local vertical deviation in mosaic created from nonlinear distorted video in our previous work [16] showing direct matching process of cropped frames.

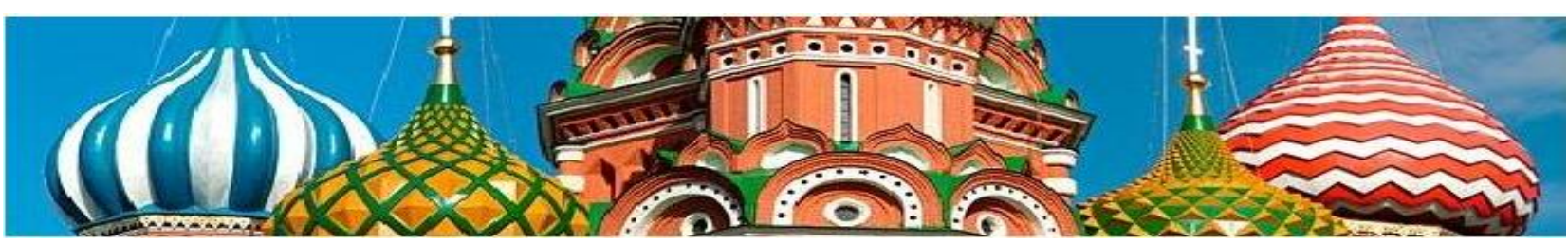

Fig. 4: Mosaic3 from video mosaicing using Strip Search Algorithm

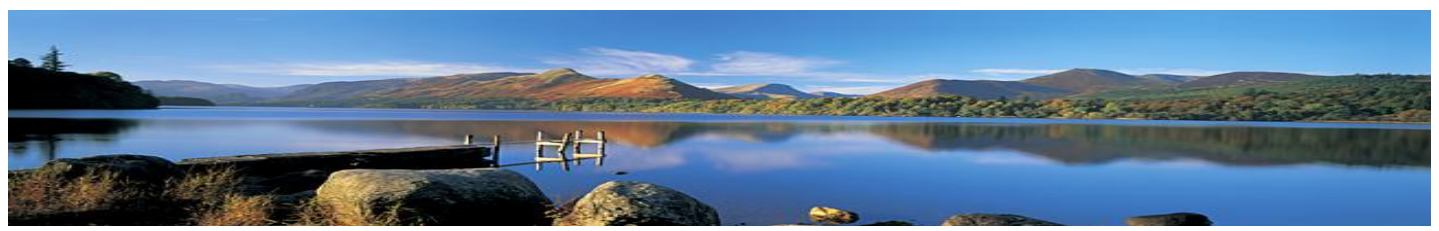

Fig. 5: Mosaic4 from video mosaicing using Strip Search Algorithm

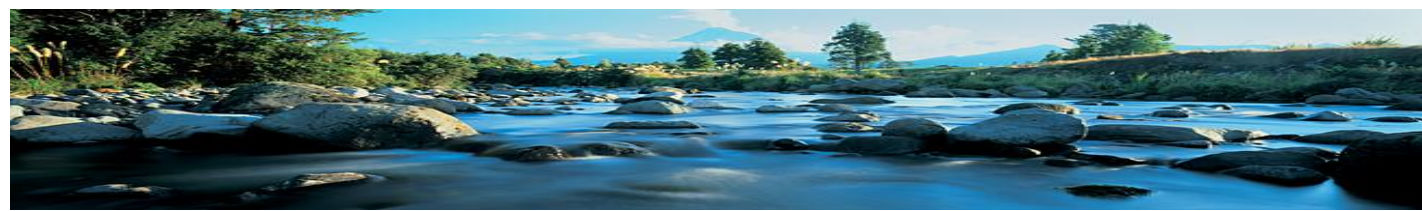

Fig. 6: Mosaic5 from video mosaicing using Strip Search Algorithm

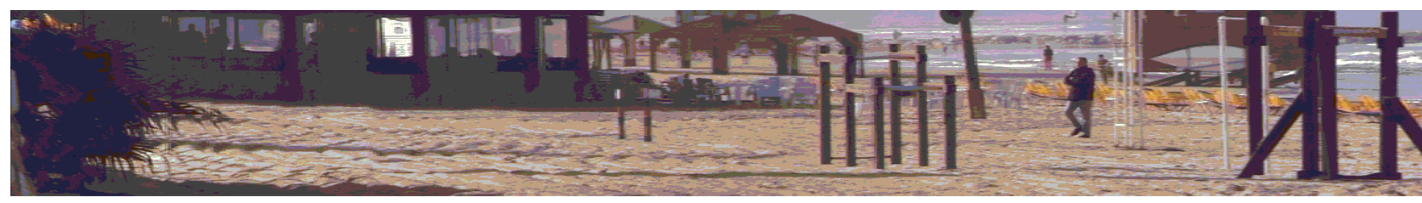

Fig. 7: Mosaic6 from video mosaicing using Strip Search Algorithm 


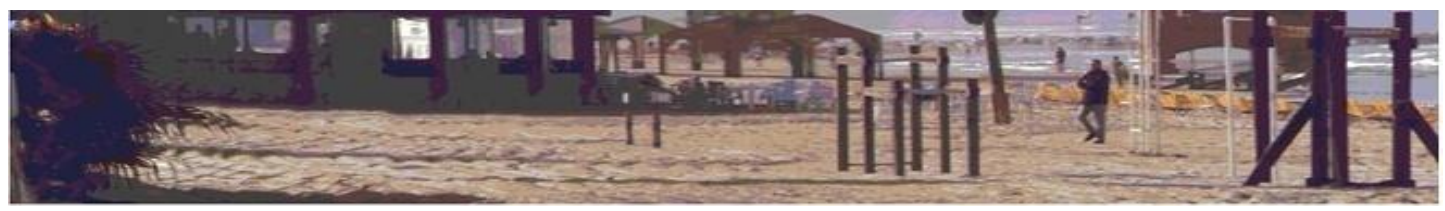

Fig. 8: Mosaic7 from video mosaicing using Wexler's method

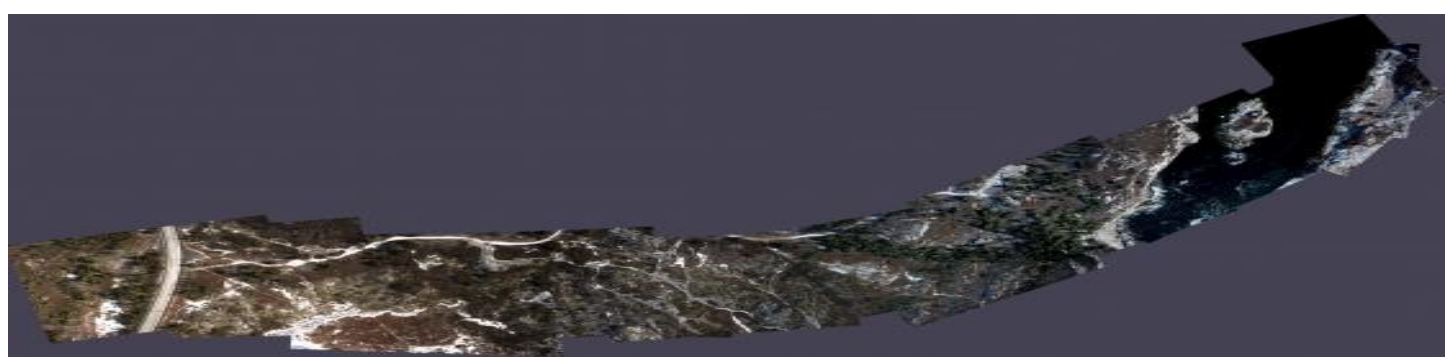

Fig. 9: Mosaic8 from image mosaicing using Andrew's method

\section{CONCLUSION}

Performance evaluation is the final and necessary step during the development phase of any technology. Earlier very few methods were presented to evaluate the performance of image/video mosaicing techniques in literature. Formulation for closeness and residue between the actual frames and the estimated frames from mosaic image is performed. Closeness and residue provides unique measure for the mosaic image with reference to the information available in video frames. It doesn't use the standard ground truth dataset. Proposed method is robust as it can be applicable to any mosaic method. This method can evaluate the mosaic results from different algorithms. This method can evaluate the performance of image mosaicing technique as well as video mosaicing techniques with nonlinear distortions by slight modifications.

\section{REFERENCES}

[1] R. Marzotto, A. Fusiello, V. Murino, "High resolution video mosaicing with global Alignment," In Computer Vision and Pattern Recognition, volume 1, pages I692-I-698, 2004.

[2] Azzari P, Stefano L. D, and Mattoccia S, "An evaluation methodology for image mosaicing algorithms.," Proceedings ACIVS, Juan-les-Pins, France, pp. 89-100, 2008.

[3] Brown M, "Multi-image matching using invariant features," Ph.D. thesis, University of British Columbia, Vancouver, BC, pp. 74-87, 2005.

[4] Boutellier J, Silvén O, Tico M, Korhonen L, "Objective evaluation of image mosaics," Commun. Comp. Info. Sci. vol: 21, 107-117, 2009.

[5] Birgit Möller, Rafel Garcia, Stefan Posch, "Towards objective quality assessment of image registration results," In Proceedings of the 2nd International Conference on Computer Vision Theory and Applications, VISAPP, pp: 233-240, 2007.
[6] Vladimir Petrovi'c, Costas Xydeas, "Objective image fusion performance characterization, "In Proceedings of the Tenth IEEE International Conference on Computer Vision, vol. 2, pp: 1866-1871, 2005.

[7] Li-hui Zou, Jie Chen, Juan Zhang, "Assessment approach for image mosaicing algorithms," Opt. Eng. Vol. 50(11), Nov 2011.

[8] Alexander Behrens, Michael Bommes, Sebastian Gross, Til Aach, "Image Quality Assessment of Endoscopic Panorama Images," Proceedings of IEEE International Conference on Image Processing-ICIP, 2011.

[9] Christopher Coffin, Sehwan Kim, Tobias Hollerer, "Evaluation of four methods for real time panorama acquisition," Phd thesis, University of California, Santa Barbara.

[10] Pierre Désaulniers, Simon Thibault, "Performance evaluation of panoramic electro-optic imagers using the TOD method," Infrared Imaging Systems: Design, Analysis, Modeling, and Testing XXII, Proc. of SPIE, Vol. 801409, 2011.

[11] Rand Shaker Al Ani, "An evaluation of the Film-Based and Digital Panoramic Radiographic Quality," Al Rafidain Dent J, vol. 8(1), pp: 67-71, 2008.

[12] Dove SB, McDavid WD, Welander U, Tronje G, "Preliminary evaluation of a digital system for rotational panoramic radiography ," Oral Surg Oral Med Oral Pathol, vol. 73(5), pp:623-32, May1992.

[13] Ladeirua DB, Cruz AD, Almeida SM, Bóscolo FN. , "Evaluation of the panoramic image formation in different anatomic positions," Braz Den Journal, vol. 21(5), pp: 458-62, 2010.

[14] ] Andrzej Glowacz, Michal Grega, Piotr Romaniak, and Mikołaj Leszczuk, "Quality Evaluation of Compressed Panoramic Images Constructed using MPEG-7-Based Image Registration," Next Generation Internet Networks, 2008. NGI 2008, pp: $167-172$. 
[15] Igor Potucek, Vitezslav Beran, Stanislav Sumec and Pavel Zemcik, "Evaluation and comparison tracking methods using meeting omnidirectional images,"pp:315,2007 .

[16] Kantilal P.Rane, S.G.Bhirud, "Image Mosaicing with Strip Search Algorithm based on a Novel Similarity Measure," International Journal of Computer Applications vol.27 (2), pp: 42-47, August 2011.
[17] Benedict Brown, Philip Shilane, "Image Mosaic," http://www.cs.princeton.edu/ bjbrown/ mosaic/Benedict Brown and Philip Shilane.mht.

[18] Wexler, Y. Simakov, "Space-time manifolds," Tenth IEEE International Conference on Computer Vision, vol: 1, pp: 858-863, 2005.

[19] Andrew Hazelden, "Andrrew Hazelden's Blog: A blog about photography, visual effects, and electronics," http://www.andrewhazelden.com/blog/auther/andrew/CH DK.mht.

Table 1: Evaluation of mosaic image

\begin{tabular}{|c|c|c|c|}
\hline Sr. No. & Algorithms & Mosaics & OPC (\%) \\
\hline 1 & Strip Search Algorithm for video mosaicing [16] & Mosaic3 as shown in Fig. 4 & 45.1730 \\
\hline 2 & Strip Search Algorithm for video mosaicing & Mosaic4 as shown in Fig. 5 & 59.5000 \\
\hline 3 & Strip Search Algorithm for video mosaicing & Mosaic5 as shown in Fig. 6 & 59.7451 \\
\hline 4 & $\begin{array}{c}\text { Strip Search Algorithm for video mosaicing (vertical } \\
\text { distortion) with full frames }\end{array}$ & Mosaic2 as shown in Fig. 3 & 32.1469 \\
\hline 5 & $\begin{array}{c}\text { Strip Search Algorithm for video mosaicing (vertical } \\
\text { distortion) with cropped frames }\end{array}$ & Mosaic2 as shown in Fig. 3 & 57.7916 \\
\hline 6 & $\begin{array}{c}\text { Benedict's method for video mosaicing(vertical } \\
\text { distortion) with cropped frames [17] }\end{array}$ & Mosaic1 as shown in Fig. 2 & 40.1676 \\
\hline 7 & $\begin{array}{c}\text { Benedict's method for video mosaicing(vertical } \\
\text { distortion) with cropped frames }\end{array}$ & Mosaic1 as shown in Fig. 2 & 58.2810 \\
\hline 7 & Strip Search Algorithm for video mosaicing & Mosaic6 as shown in Fig. 7 & 59.7871 \\
\hline 8 & Wexler's method for video mosaicing [18] & Mosaic7 as shown in Fig. 8 & 59.4314 \\
\hline 9 & Andrew's method for image mosaicing [19] & Mosaic8 as shown in Fig. 9 & 54.5462 \\
\hline
\end{tabular}

